\title{
Journal article \\ Performance comparison of geo-referencing a radar using prism method with global positioning system
}

\author{
Ebenhezer Mabotha ${ }^{1}$ and Nkateko Mabunda ${ }^{2}$ \\ 1 University of Johannesburg; emabotha@gmail.com \\ 2 University of Johannesburg; nkatekom@uj.ac.za \\ emabotha@gmail.com ; Tel.: +27-78-863-0009
}

\begin{abstract}
Monitoring of the surface operations using movement and surveying radar (MSR) can prevent loss of life, equipment, production and loss of the mine. Slope monitoring using MSR is an important aspect of open-pit mining as it provides real-time movement of deformation data for the slope. It is therefore important that the radar is accurately geo-referenced in order to provide accurate real-time movement data. Georeferencing is defined as the process of determining an instrument's position (in the form of Easting, Northing, Height) as well as the orientation with respect to the mine's local coordinate system. This helps in getting georeferenced data points from the radar that are identified by a unique set of coordinates in relation to the mine's coordinate system which allows the radar to track movement for a specific set of coordinates. In this research, we assess the performance of geo-referencing a radar using the total station method and compare it with the integration of Advance Navigation - Spatial Dual GPS system connected via RS422 on the MSR. This includes usage of the Spatial Dual navigation coordinates output to calculate the radar's position relative to the mine local coordinates and mapping the radar's azimuth, elevation and Range (Az, El and $\mathrm{Rl}$ ) values to the measured pit-slope data points. Furthermore, a comparison of key attributes of both methods of georeferencing is performed using a matrix system and giving an overall performance appraisal of both systems. Integrating a navigation system allows the radar to have an auto geo-referencing functionality that will reduce the time spent in completing this process. The findings reveal that the GPS obtained a higher score than the total station with prism method on the weighted matrix system. The total station was found to be more accurate than the GPS however, the deployment time for the GPS is quicker than that of the total station. This is important for different operation such as strip and open-pit mining to choose the preferred method of georeferencing depending on the level of accuracy required.
\end{abstract}

Keywords: Geo-referencing; Surveying radar; Mining; Global positioning system; Slope Monitoring;

\section{Introduction}

In open-pit and strip mining, activities such as blasting causes strain and stress on the wall around the pit. This may result in slope failure, which is a risk factor for both business and safety of the mine. Slope failure can have critical consequences such as injury to personnel, loss of lives, loss of production and damage to mining equipment. It is therefore imperative that the slope is monitored for potential failures [1]. Slope monitoring is important as it serves as a warning system for mine personnel of any areas on the pit slope that are unstable. An example of a slope-monitoring tool in open pit mining is the movement and surveying radar (MSR) developed by Reutech Radar Systems [2]. This provides real time movement data with precise coordinates of each point measured. A process called geo-referencing achieves the determination of an individual coordinate point. Mining operations are usually large and typically utilize their own site-specific coordinate system that is different from the traditional and main stream coordinate systems applied. Because of the differing coordinate systems, whenever a MSR is deployed, there is a need to geo-reference it to ensure that the data collected can be allocated to the correct Easting, Northing and Height (X, Y and Z) location on the pit slope. Geo-referencing is a process of aligning geographic data to a known coordinate system so it can be viewed, queried and analyzed with other geographic data [3]. In simple terms, it is used to assign the location of a device or point to a system of geographic coordinates so that the location or point is apportioned to its real world location. When applying radar technology to pit-slope monitoring, geo-referencing is paramount in 
determining the exact location or coordinates for points and portions of the pit slope that are moving in order to mitigate pit slope instability and potential failure.

\section{Similar studies}

Previous studies related to geo-referencing and surveying shows that positioning can be acquired using the total station with prisms and the use of GPS. The operation accuracy of total station and prisms are discussed in details by [4] and [5] respectively. These studies proved in details the factors that affect the accuracy of the total station which includes: temperature, humidity and pressure as well as the interruptions between the beam path.

The GPS accuracy and application of the different types is discussed in detail by [6]. This is important for this field of study as it clearly gives the best choice for application in geo-referencing or positioning in general. Acquiring of position of GPS in 2D and 3D is discussed in detail by [7]. This also aims at giving factors such as multi-path that can affect the accuracy of the GPS.

\subsection{Application of movement and surveying radar (MSR) in slope monitoring}

MSR is used to measure the stability of the pit slope [2]. In principle, radar systems operates by radiating out a sinusoidal electromagnetic wave towards the target and detect the electromagnetic wave reflected back from the targets. According to [8], radar is all about waves to detect the presence of an object and to find their position. The reflected signal will scatter off any object that it encounters and some of that signal will be reflected back $180^{\circ}$ out of phase. The reflected signal is then compared with the transmitted signal to determine the properties of the target.

The reflected wave is captured by the antenna and routed via the circulator to the receiver. The receiver amplifies the reflected signal and route it to the analogue to digital converter (ADC). The converted values are then fed to the computer, which will display the target information on the HMI. The target information displayed on the plane can be viewed according to the mine's coordinate system. This can be done only if the radar is geo-referenced. The returned signal from the target can be assigned X, Y, and Z coordinates. The accuracy of the point will depend on the accuracy of the radar's geo-referenced position.

In mining application, MSR is used to measure the phase and amplitude of the reflected signal for up to a distance of $4 \mathrm{~km}$ [2]. As the radar moves the antenna to scan the azimuth and elevation, different parts of the slope are illuminated by a narrow torch-like beam. These parts of the slope will then reflect a radio frequency energy and this can be used to determine the displacement of the slope over time. The slope movement or changes in the relative range can be determined from the phase of the radar signal. The range to the slope can be calculated using the distance formula (1)[9].

$$
\mathrm{R}=\frac{c \times t}{2}
$$

The speed of a radar wave is equal to the speed of light and therefore : $c=3 \times 10^{8}$ [9].t $=$ time taken to receive the return signal. The range is halved because the signal travel to and from the slope. Should the slope move, the next measured return signal of that point changes in phase-shift.

The following radar measurement are the used to calculate the phase. The Radar uses the phase shift difference between the two measurements to determine the relative range. The MSR uses a frequency of approximately $10 \mathrm{GHz}$ in order to meet the required precision wanted in mining application. MSR utilizes a wavelength of $30 \mathrm{~mm}$ and therefore a phase shift of $5^{\circ}$ will be equal to $0.2 \mathrm{~mm}$ of movement as expressed in Formula 2. The phase shift is converted from degree to millimeters so that they can be easily interpretable for 
movement accumulation. The MSR will then accumulate these phase shift changes in relative range to track the movement of the pit slope over time.

$$
\text { Phase Shift }=\left(\frac{5}{360}\right) \times\left(\frac{\lambda}{2}\right)=0.2 \mathrm{~mm}
$$

As the radar antenna moves and receives the return signal from the pit slope, the center point of each beam is plotted on a plane. These center points are used to visualize the pit slope using the human machine interface (HMI) and they can help in determining the stability of the wall over time [2]. This is shown by using different colorings based on the level of deformation of each point over time. The MSR overlaps the beam of the signal and each center point is plotted.

Each center point of the beam can be allocated the Azimuth, Elevation and Range by the radar encoders and amplitude measurements. Once the radar is geo-referenced to known mine beacons, the Azimuth Elevations and Range of the center points can be allocated the Easting, Northing and Height coordinates based on the local mine coordinate system. This helps the mine personnel to know precisely where the deformation and potential failure of the pit slope will occur. Without geo-referencing the MSR, the data obtained will not be referenced and therefore it will be difficult to determine were the deformation is occurring.

\subsection{Geo-referencing and Geo-referencing process}

Geo-referencing can be seen as an overall term for describing techniques that are used to uniquely identify geographical objects [10]. Any point on the surface of the earth can be represented in the form of latitude, longitude and the height, which is above sea level. These points are sometimes referred to as geographical, spherical or geodetic coordinates. These types of coordinate systems can be represented on a Rectangular Cartesian system in either two or three dimension [11].

MSRs use the above mentioned coordinate system to determine the radar's position and to give more defining data from the system. This is combined with the Total station's azimuth and elevation to give the orientation of the system when geo-referenced.

When using the current manual method of geo-referencing the radar, the mine will collect and provide reference points as targets. These points are manually collected by the surveyors and can sometimes move due to vibrations and wall instability. This process is described in detail by [12]. Using these reference target, georeferencing can be completed using the total station and prisms [2]. The radar is equipped with a data processor that uses mathematical algorithms, which employ measurements from the total station to establish where it is situated relative to the mine's coordinate system. It is important to note that there are two prism mount points on the radar that have references (left and right) and their positions are known relative to the radar's center point (gimbal). This allows the orientation of the radar to be determined [2].

\subsection{Accuracy of geo-referencing using total station}

The accuracy of geo-referencing a radar using total station is mainly be determined by the accuracy of the total station, prisms [13] and the accuracy of the points that are collected by the user. The current total stations used by Reutech are Leica TS03 and TS07 which can manually be adjusted to point the target. According to Leica geosystems recommendation (Leica Geosystems), in order for the total station to give accurate reading and precise measurements it is important to constantly calibrate and operate the system within the manufacturer specifications.

Standard GNSS system provides a position accuracy of $2.5 \mathrm{~m}$ but some advance GPS can provide an average horizontal accuracy of up to $\pm 10 \mathrm{~mm}$ using triple frequency RTK GNSS receiver as well as differential GPS method. [14] gave a full classification of several GNSS positioning system types and described how each type works. This gives a more in depth understanding of this concept. 
A specifications in [13] revealed that the total station accuracy is $\pm 5 \mathrm{~mm}$ around each axis ( $\mathrm{X}, \mathrm{Y}$, and $\mathrm{Z}$ ). Total stations accuracy is affected by humidity, temperature and pressure but this can be corrected for atmospheric changes by inputting changes into the parameters [13]. Beam interruptions and moving objects within the beam path can results in the inaccuracy of the reading thereby creating false readings. It is therefore important that there is a clear line of sight and adjusted before measurement. Errors associated with the total station can be reduced or removed with two-face measurement. [15] has illustrated errors affecting the horizontal and vertical measurements, and how to adjust them. It is also important to note that the type of prisms used during measurements may cause some deviations and therefore it is important that Leica recommended prisms be used [13]. Accuracy of standard prism distance measure may vary depending on the type of prism used. [15] details the accuracy of measurements to standard prisms.

\subsection{Global positioning system (GPS)}

Global positioning system was developed by the department of defense in the USA to provide the US Forces with a more reliable, navigation positioning ability wherever they were. The system consist of multiple satellites, which revolves around the earth, which allows access worldwide, and free. A GPS calculates its position by measuring the distance from different satellites. This is accomplished by calculating how long the radio signal takes from the satellite to the receiver and multiplying the time by the speed of light [6]. Positioning is accomplished by using the signal that is transmitted by satellites to ground receivers. Using multiple receivers simultaneously in a differential mode relative position can be measured between them and can be done so with high accuracy of a few millimeters [6][7].

In mining applications, high levels of accuracy are necessary. Standard GPS accuracy is low and can only be used in applications of low accuracy of positioning as discussed by [16]. To achieve better accuracy as required in the mining industry, the use of relative positioning technique such as a differential GPS is necessary. This can also be integrated with the use of Real-Time Kinematic (RTK) systems for an accuracy of a few millimeters as described by [6][7].

To use the GPS for positioning, the receivers require an unobstructed view of at least four satellites. For open pit mining this is possible because the operation is on the surface and therefore there is always unobstructed view to most satellites. The GPS uses three satellites to determine the horizontal position (2D) and the fourth satellite is used to determine the altitude. The accuracy of the GPS can be affected by a concept called multipath as described by [7].

\subsection{Contribution to the study}

Although total station and GPS have been used in the mining industry for geo-referencing and tracking, this research has its own importance. The precision of individual systems is clearly illustrated by [13], [15] and [17]. Integrating the GPS on the movement and surveying radar gives the MSR auto geo-referencing capabilities that will enhance the speed of deployment in the field. In this research, the steps and processes that were followed to integrate the GPS on the MSR and comparing it to the already used total station system is clearly explained. Furthermore, a matrix system was developed for comparing the two systems for geo-reference. This consists of accuracy, cost, time, accuracy and reliability attribute that are calculated from the performance of the systems and therefore results in an unbiased comparison.

\section{Research methods}




\subsection{Introduction}

This is an experimental research. Several experiments were carried out to perform comparison relevant to integrate the GPS on the MSR. The integration of the GPS and antennas are done on an already existing system. Figure 1 shows the hardware connection on the MSR and illustrates the integration of the Advance navigation system that will be used for comparison.

\subsection{Hardware architecture}

Figure 1 shows the block diagram of the integration of the GPS on the MSR. The Advance Navigation GPS uses the Optical channel data unit (ODU) Mini snap Series B connector for communication and power supply. The power source is not from the communication device and therefore the cable was tapped and split into two to accommodate this. Pin 2 (Power) and Pin 9 (Power ground) of the connector are for power and the rest are connection to the serial to Ethernet converter. The GPS system supports voltage range of 9V -36V [17] and therefore is supplied with $24 \mathrm{~V}$ from the existing radar power supply. The serial to Ethernet converter will pass the information to the main Ethernet switch and the switch will pass the information to the HMI laptop.

The Advance Navigation - Spatial Dual GPS system was chosen for this experiment as it can provide accuracy of up to $8 \mathrm{~mm}$ horizontal (with RTK) and $0.5 \mathrm{~m}$ vertical accuracy. This system provides accurate position and orientation in difficult conditions as suggested by [17] and therefore it will be suitable for this application. The system also allows two antennas to be used and therefore will allow direction finding using multiple antennas as discussed by [18]. This method of direction finding was chosen because the radar is always stationary.

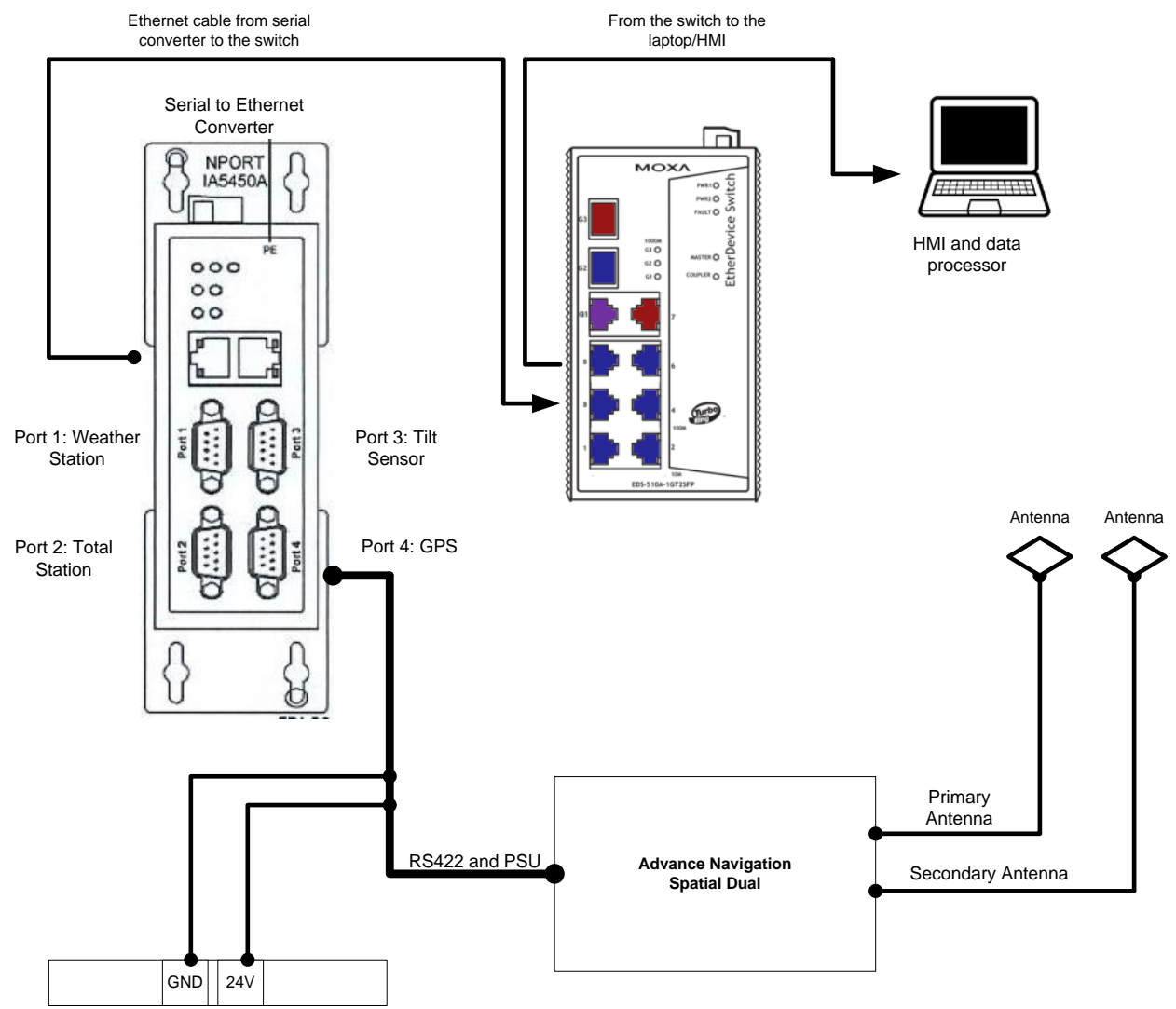

Figure 1: MSR GPS integration diagram

\section{a. GPS fitting mounting}

The GPS device was fitted on the existing signal data processor chassis plate. Figure 2 illustrates the GPS device mounted on the bracket and also on the chassis place inside the radar. 

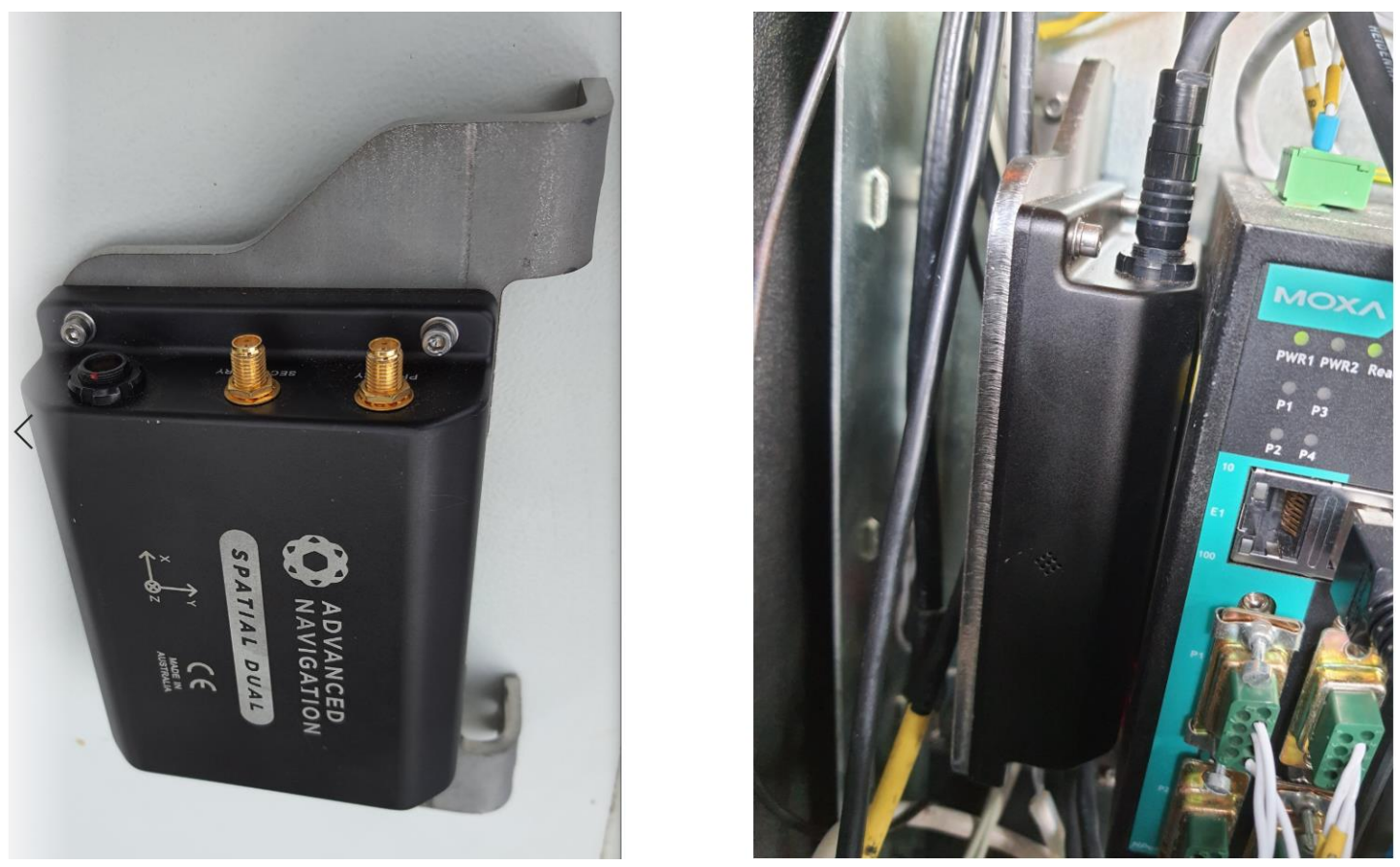

Figure 2: GPS mounted on a bracket (Left picture) and installed on the MSR (Right picture).

\section{b. Antenna mounting}

Two antennas with a baseline of $1 \mathrm{~m}$ were used for direction finding. According to [17], a baseline of $1 \mathrm{~m}$ will give a heading accuracy of 0.1 degree. The antenna baseline is shown in Figure 4.
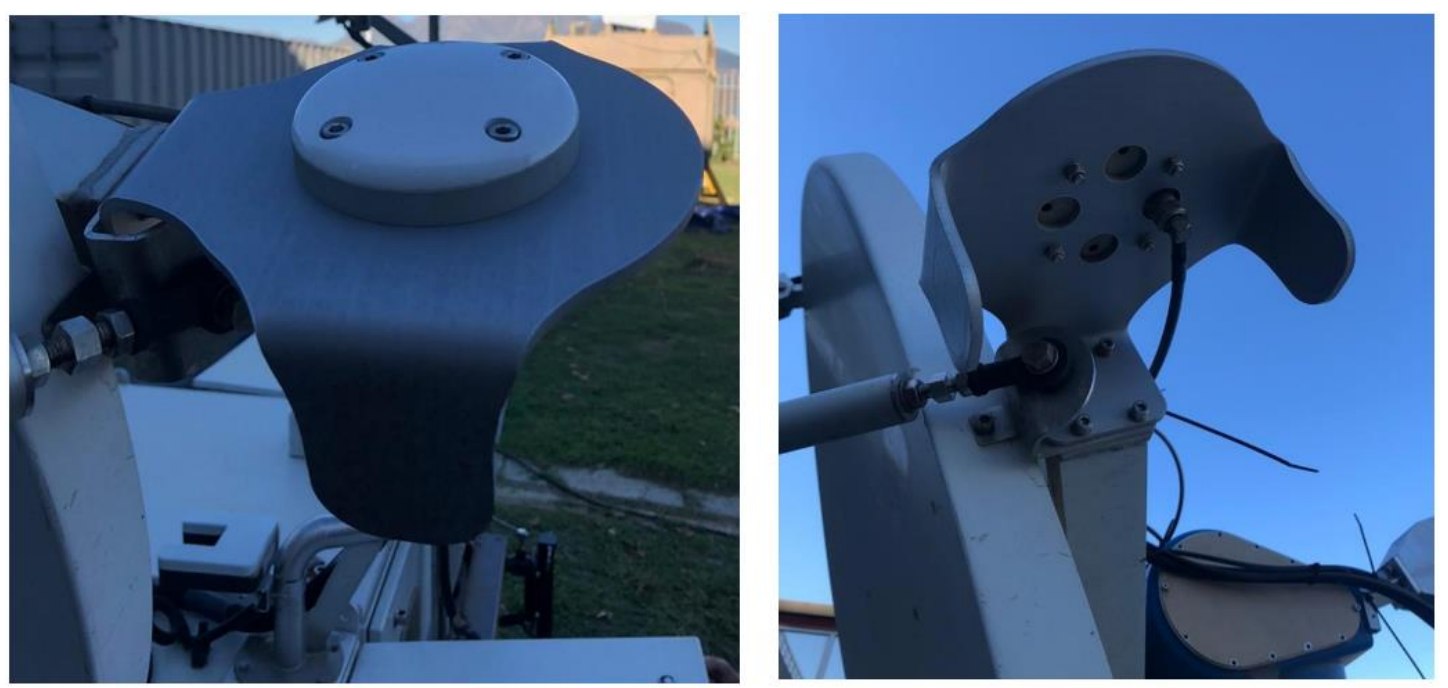

Figure 3: GPS antennas mounted on the MSR antenna. (Advance Navigation, 2017). 


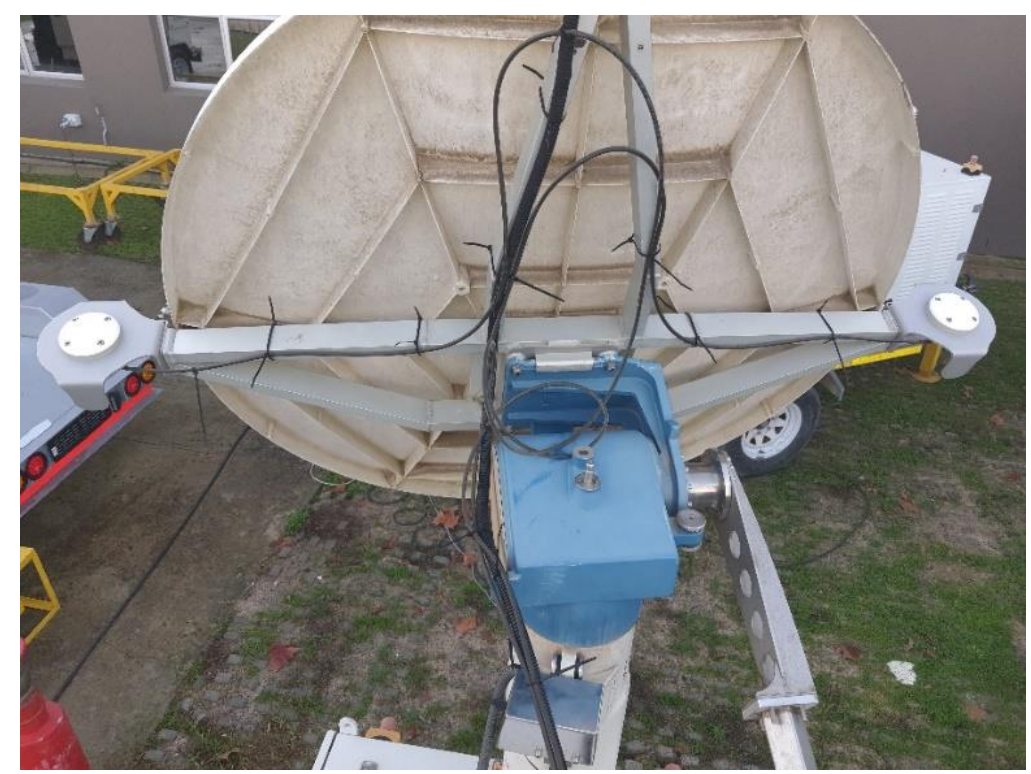

Figure 4: Antennas installed on the MSR with a baseline of $1 \mathrm{~m}$

\section{c. GPS adjustments and offsets}

In order to integrate the GPS on the MSR, the following adjustments and offsets were configured. These adjustments mainly depend on the position and orientation of the GPS as well as the alignment of the dual antennas on the MSR.

i. Alignment Offset

ii. Pitch Offset $=90.000$

- X Offset: 0.3

- Y Offset: -0.5

- Z Offset: 1.2

iii. Dual Antenna Offset

- Automatically

- $\quad$ Primary = Left (SDP side)

- $\quad$ Secondary = Right (CP side)

The setup and adjustments were based on the instructions specified in [17].

\subsection{System control software}

The system control software (SCS) is used to process the scan information, manage and control the functionality of the radar. In order to interact with the GPS it was modified to send the following packets to the spatial dual navigation GPS :

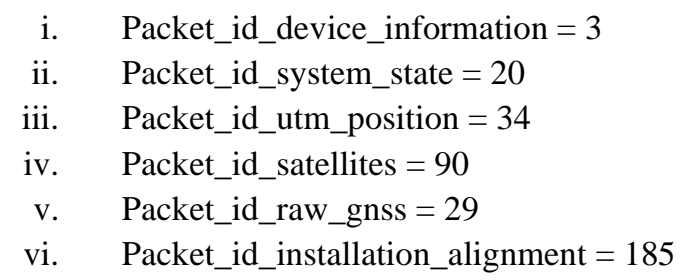

The above packets are sent on start-up and then wait for the GPS device to start streaming the requested information on the port specified during configuration. Since the configuration is done on start-up the SCS then 
continuously checks if there is available information on the port and then process it accordingly. After processing the streamed information, the results were used to calculate the position of the GPS relative to mine coordinates system. In order for the software to know the coordinate system that will be used, the user will be required to enter at least four (3) reference points that which includes the GPS coordinates and corresponding local coordinates. The position and heading of the radar is then calculated using advance algorithm. The results of the GPS calculation and offset needs to correlate with the local coordinate system, otherwise geo-referencing will fail. The software will continuously request the position from the GPS until the system is georeferenced. Figure 5 illustrates the software flow diagram.

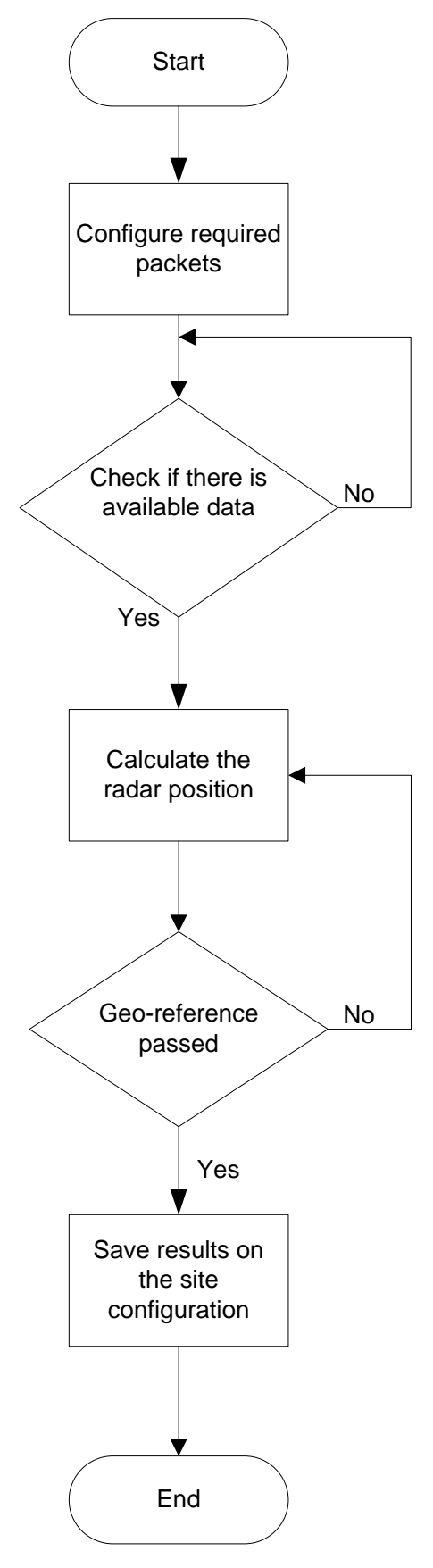

Figure 5: Geo-referencing software flow diagram

\subsection{Data collection and matrix system}

Collected data was stored as the comma separated values (CSV) files, they were generated by the Spatialdual GPS that stored and deploy them in this format. GPS geo-referencing measurements were obtained using 
serial communication to the GPS vial RS422 and stored in CSV format. Validation of the results was also done using Microsoft excel and comparing it to the pictures from generated by the Spatial-dual GPS. The results from the comparison were recorded on Microsoft Excel 2016 order to compare the expected/actual position and obtained ones. The proposed matrix system table illustrated in Table $\mathbf{1}$ was used to record and scale the performance of the individual geo-referencing method. The matrix table consists of a variety of parameters, which are described in the proceedings sections.

Table 1: Matrix system template for geo-referencing

\begin{tabular}{|l|c|c|c|}
\hline Decision Matrix & \multicolumn{2}{|c|}{ Options } & Weight \\
\hline Criteria & $\begin{array}{l}\text { Total station } \\
\text { Method }\end{array}$ & Automated GPS Method & \\
\hline Accuracy & 0 & 0 & $40 \%$ \\
\hline Cost & 0 & 0 & $20 \%$ \\
\hline Time & 0 & 0 & $20 \%$ \\
\hline Effort & 0 & 0 & $10 \%$ \\
\hline Reliability & 0 & 0 & $10 \%$ \\
\hline Total & & & \\
\hline Average weight for TS Method & $\mathbf{0}$ & \\
\hline $\begin{array}{l}\text { Average weight for GPS } \\
\text { Method }\end{array}$ & $\mathbf{0}$ & \\
\hline
\end{tabular}

In order to determine the input values for the matrix system, performance comparison was carried out and the results were recorded comparison table. The comparison tables are used to calculate the difference between the actual surveyed position and the obtained point from either the total station method or the GPS method. The matrix system consists of a variety of parameters, which are described in the proceedings sections.

\section{a. Accuracy}

This is the closeness of the measurement to the true value. This can be calculated using RMS and standard deviation. On every deployment, the obtained results are compared with the expected values. The difference is calculated and the percentage error can be determined using the formula (3).

$$
\% \text { error }=\frac{\text { error }}{\text { actual value }} \times 100
$$

In order to keep the results fair, the coordinate system used to create the local coordinate system for the site was regarded as true and accurate. Therefore, the results are compared to the local coordinate system. The higher the difference from the actual value, the lower the accuracy value. The accuracy of the coordinates measured was determined using the scale in Table 2. The accuracy and precision of the data measurements is determined using the RMS and standard deviation on the data collected. Root mean square is the measure of accuracy of the individual measurement. It can be calculated from the deviation between the true and measured points.

Table 2: Accuracy measurement table

\begin{tabular}{|c|c|}
\hline Scale & Value \\
\hline $0 m-0.5 m$ & 5 \\
\hline $0.5 m-1 m$ & 4 \\
\hline $1 m-2 m$ & 3 \\
\hline $2 m-5 m$ & 2 \\
\hline $5 m+$ & 1 \\
\hline
\end{tabular}




\section{b. Equipment cost}

The cost of both system plays an important role in determining the best technology to use. Some operations may require the best accuracy and would not mind the deployment time and some may not require the best accuracy but care more about the deployment time. Therefore, this component may vary from operation to operation. The actual cost of each system will be made available to illustrate the difference. The cost of each system are only be stated in terms of hardware and it excludes the customization and algorithm to integrate with the MSR. The percentage difference of the costs are used to input on the matrix table to determine the cost score. The system with the lowest price will score the maximum score. The percentage difference between the systems will be used to score the more expensive system.

The percentage difference can be calculated using formula (4):

$$
\% \text { diff }=100 \%-\frac{\text { Lowest price }}{\text { Highest prices }} \times 100
$$

The percentage table shown in demonstrates the matrix score of the difference.

\section{Table 3: System cost difference from the cheapest}

\begin{tabular}{|c|r|}
\hline \% Difference & Value \\
\hline $0-20$ & 4 \\
\hline $20-40$ & 3 \\
\hline $40-60$ & 2 \\
\hline $60+$ & 1 \\
\hline
\end{tabular}

\section{c. Deployment time}

Deployment time is the time it takes for a user to geo-reference the radar. This criteria is based on the scale shown in Table 4.

Table 4: Deployment time scale

\begin{tabular}{|c|c|}
\hline Time Scale & Value \\
\hline $0-10 \mathrm{~min}$ & 5 \\
\hline $10-20 \mathrm{~min}$ & 4 \\
\hline $20-30 \mathrm{~min}$ & 3 \\
\hline $30-40 \mathrm{~m}$ & 2 \\
\hline 40min and more & 1 \\
\hline
\end{tabular}

\section{d. Effort}

Effort is the amount of work and personnel required to complete geo-reference. It can also be defined as the use of physical or mental energy of trying to do something. This may differ from user to user and therefore the component is based on user's discretion. The score for this component is between one (1) and five (5).

\section{e. Reliability}

Reliability can be seen as the amount of time that the system can be deployed without failing. This is important for this test because the number of times that a system can be deployed without failing contributes to it overall performance. The reliability percentage can be evaluated by using formula (5):

$$
\begin{gathered}
\text { Reliability }(\%)= \\
\frac{\text { Number of deployments }- \text { Number of failed attempts }}{\text { Number of deployments }} \times 100
\end{gathered}
$$




\subsection{Setup for the test}

Figure 6 illustrates the setup of the radar with the GPS/Total station and the corner reflector. The GPS was connected to the existing serial interface of the MSR. The corner reflector was chosen as a reference target for every deployment because it is a good signal reflector. The radar was geo-referenced using both methods, where for each method the corner reflector was scanned. The center of the corner reflector is the point that gives the highest return, therefore this point's coordinates were taken as a reference point. This way of performing the comparison of the methods does not only help us determine the radar position but it helps in making sure that the target is also located correctly.

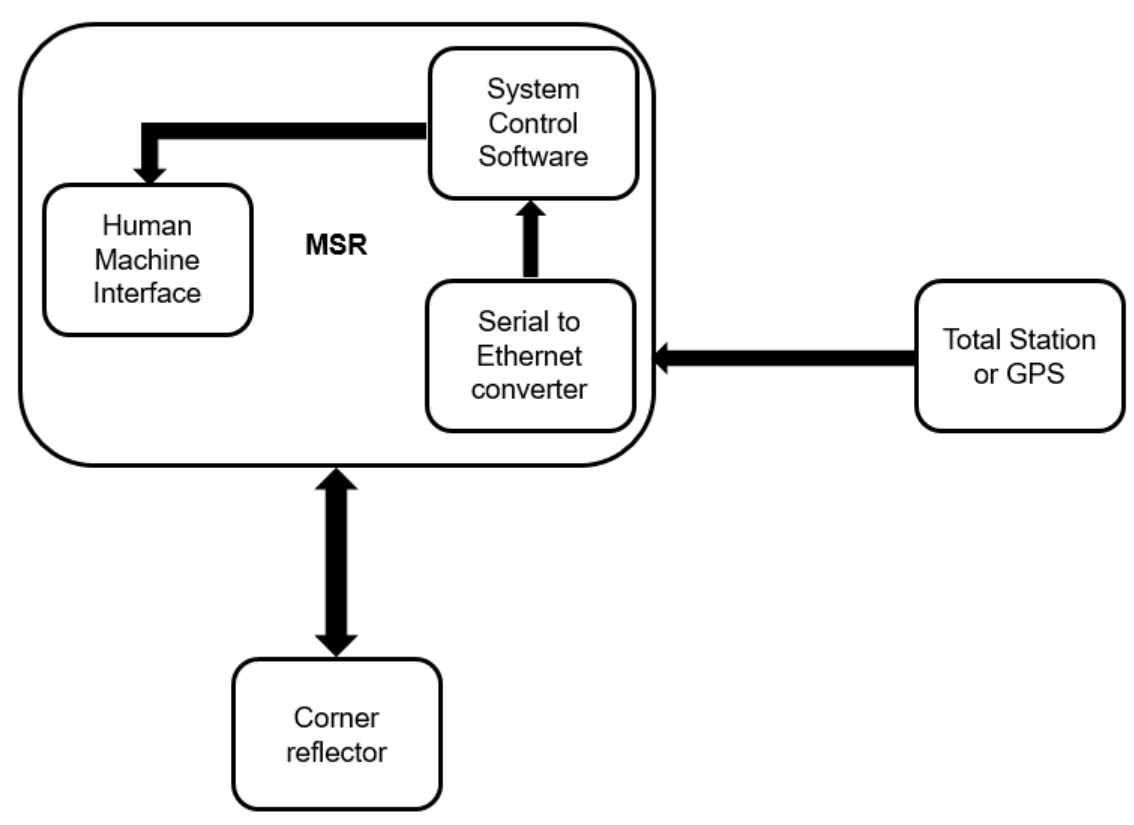

Figure 6: Hardware setup block diagram

\subsection{Deployment and comparison}

In order to compare the GPS and total station method, the coordinates of the center of the corner reflector were recorded using the system that was used to derive the local coordinates. These coordinates are regarded as the accurate. The radar is then used to scan the corner reflector and record the coordinates of the center point for each geo-referencing method. This means that each deployment will have at least two set of data, one for the GPS and one for the total station. Some deployments are repeated without changing the setup in order to verify the already acquired results and to verify reliability. In each deployment, the center of the corner reflector is verified using the amplitude return output from the MSR. A typical amplitude output from the MSR is shown in Figure 7. This shows the amplitude $(\mathrm{dB})$ and the coordinates of the selected point in the form of Easting, Northing and Height (E, N, RL). 


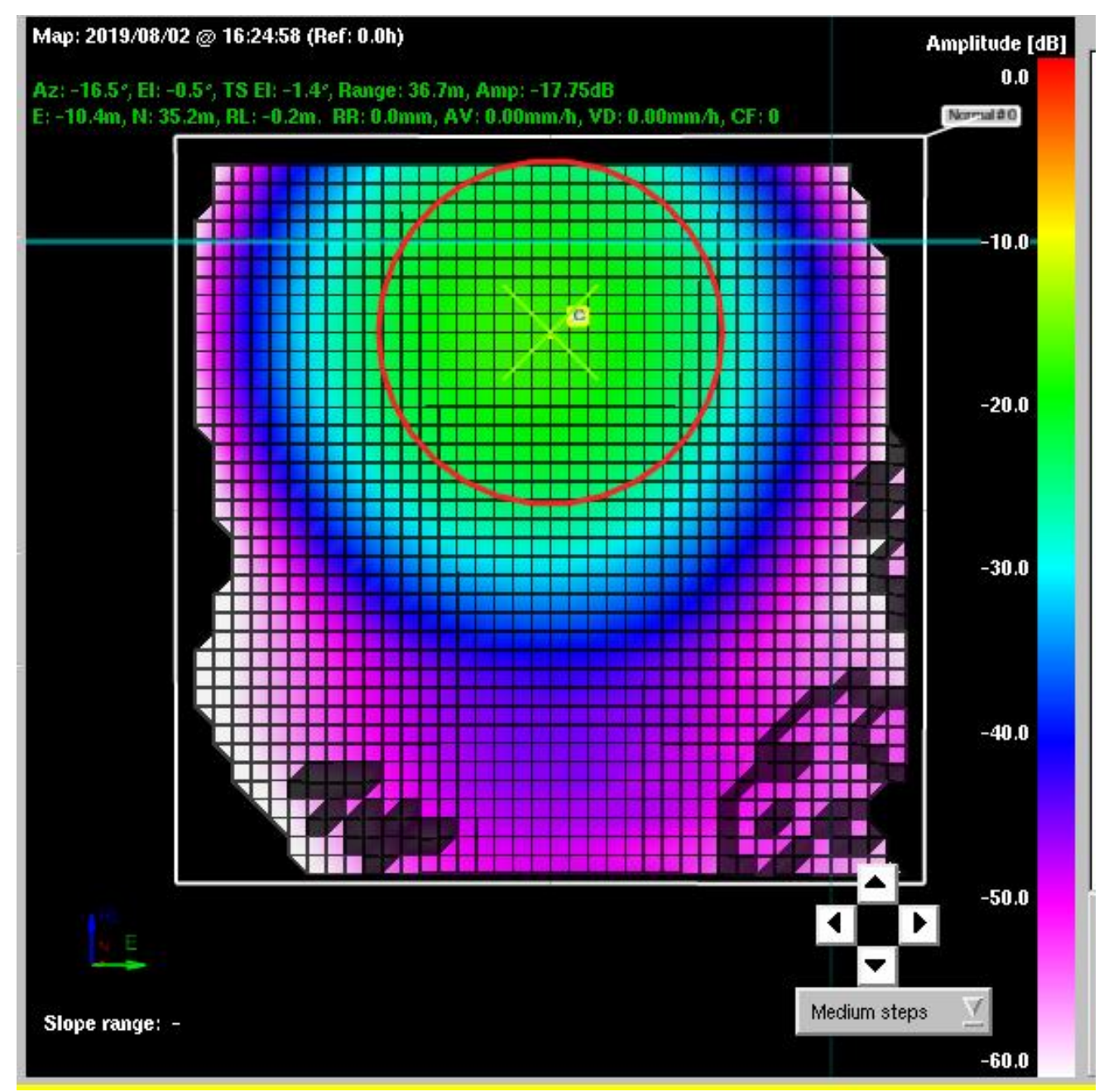

Figure 7: Corner reflector amplitude output

\subsection{Local coordinate points}

Reference points were picked locally and the beacon was used as the origin point. Table $\mathbf{5}$ shows the reference points used for the test located in Stellenbosch, Technopark. These points are regarded as true or accurate points and they represent the local coordinate system.

Table 5: Local Coordinate points

\begin{tabular}{|l|l|c|l|l|}
\hline \multicolumn{5}{|c|}{ Coordinates } \\
\hline Point No. & Description & X(North) $[\mathbf{m}]$ & Y(East) $[\mathbf{m}]$ & $\mathbf{Z}(\mathbf{U p})[\mathbf{m}]$ \\
\hline 1 & Beacon & 0 & 0 & 0.515 \\
\hline 2 & Back left corner & 76.46 & -74.51 & 1.22 \\
\hline 3 & Back right corner & 116.98 & 36.07 & -0.52 \\
\hline 4 & Rooftop RHS & 124.47 & -30.35 & 9.38 \\
\hline 5 & Rooftop back LHS & 113.84 & -57.14 & 9.44 \\
\hline
\end{tabular}

The coordinates were spread around for better accuracy and they were positioned in such a way that they will be visible to the total station when comparing the methods.

Figure 8 shows the above coordinates from an aerial view. 


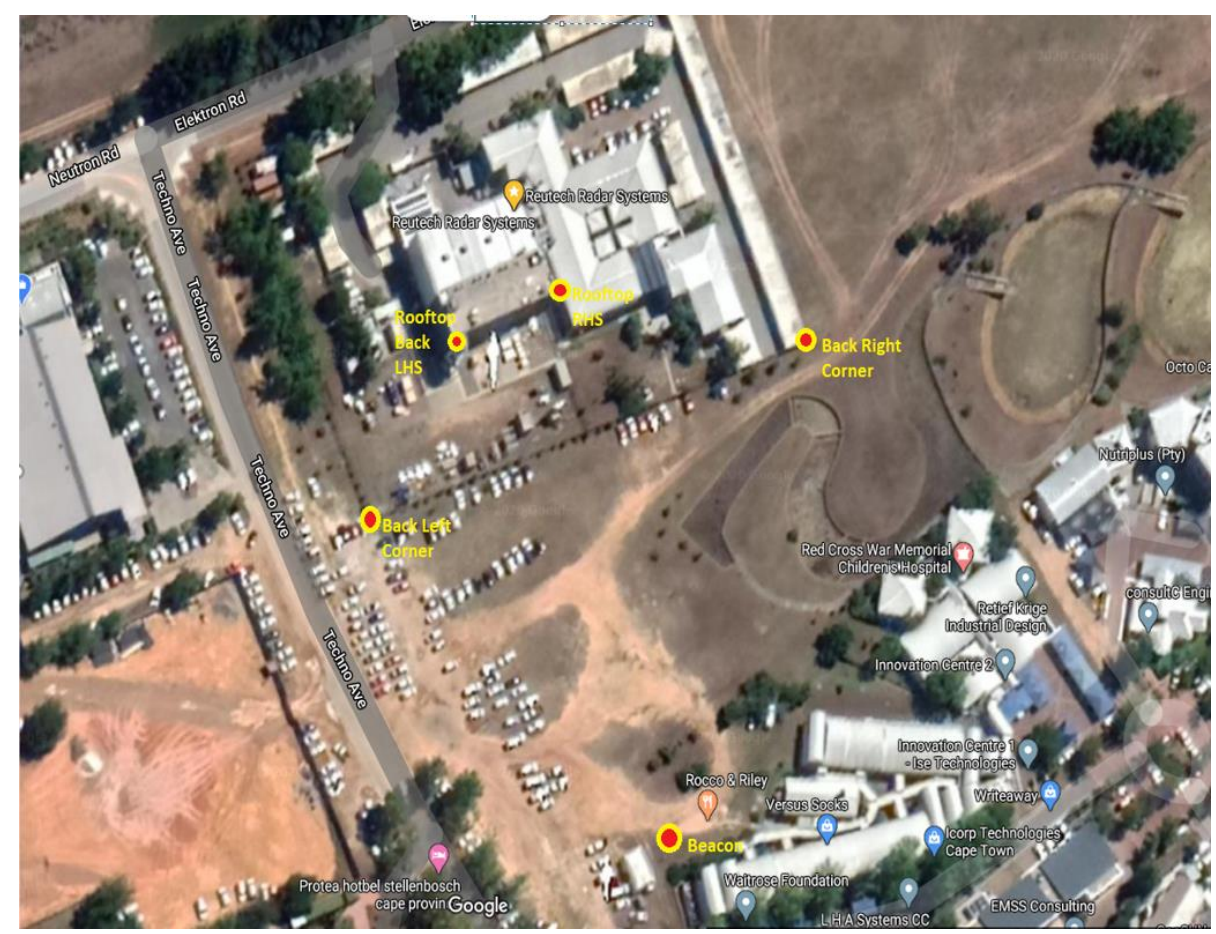

Figure 8: Local coordinate aerial view map. Map taken from google maps.

\subsection{Errors}

Measurements can be affected by different factors. During deployments and performing comparison, errors were eliminated using the following steps:

\subsubsection{Gross errors}

Gross errors are caused by human interaction such as bad weather resulting in blurry vision to the target. These type of errors can occur when using the total station while observing the reference points and prisms. They are eliminated or verified by two face measurements and being careful when recording the data. Some of the gross errors that were encountered during deployments are listed below:

- Errors capturing the deployment results into the spreadsheets

- Deviations in measurements of the reference points and prisms using the total station

\subsubsection{Instrumental errors}

Instrumental errors arise due to the deficiency of the instrument, instruments that are not calibrated and misusing of the instrument. These errors can be eliminated by doing the following:

- Calibrating the instrument

- Use the instrument as specified by the manufacture

\subsubsection{Random errors}

These errors are caused by measured data being inconsistent when the measurements are repeated. During the experiments, these errors could have been caused by human factors (senses sight and touch), instrument errors and environment. They were accounted for by performing calibration and double checking the measurement results. The variations may occur even after the gross or instrumental errors have been accounted for. Since these errors are not noticeable, they become unrecognizable and impossible to eliminate or reduce. These errors are corrected by repeating the same measurements more often. 


\section{$4 \quad$ Results and analysis}

The advance navigation's spatial dual system was chosen for this research. After studying the manufacture specification, the following configuration were set on the GPS in order to integrate it with the MSR.

\subsection{Data analysis}

After data was collected, it was organized and analyzed. The GPS test results were analyzed using Microsoft Excel 2016. Average deployment position versus time was created and the results were plotted on line graphs. Performance comparison of the total station and GPS was done using the MSR HMI. Results and graphs were also recorded on the comparison tables.

\subsection{Deployment time measurements results}

The first step in this research was to test the accuracy of the GPS. The GPS was deployed at five different sites to confirm its manufacturer's specifications. The sites and test results are listed below:

a. Stellenbosch Square

Coordinates:

\begin{tabular}{|l|l|l|l|l|}
\hline Parameter & Latitude & Longitude & Height & Heading \\
\hline Average & -33.977435586 & 18.841447770 & 152.8463 & 20.44102 \\
\hline
\end{tabular}

b. Mooiberge Farmstall parking area

Coordinates:

\begin{tabular}{|l|l|l|l|l|}
\hline Parameter & Latitude & Longitude & Height & Heading \\
\hline Average & -33.998871162 & 18.826219869 & 131.5089 & 14.90821 \\
\hline
\end{tabular}

c. Lyndoch Petrol station on Baden Powell Drive

Coordinates:

\begin{tabular}{|l|l|l|l|l|}
\hline Parameter & Latitude & Longitude & Height & Heading \\
\hline Average & -33.982219188 & 18.770455438 & 60.67923 & 43.56803 \\
\hline
\end{tabular}

d. Baden Powell Drive near Van Rhyn's distillery

Coordinates:

\begin{tabular}{|l|l|l|l|l|}
\hline Parameter & Latitude & Longitude & Height & Heading \\
\hline Average & -33.956665254 & 18.805519009 & 93.97037 & -146.715 \\
\hline
\end{tabular}

e. R44 between Dorp street and Die Boord

Coordinates:

\begin{tabular}{|l|l|l|l|l|}
\hline Parameter & Latitude & Longitude & Height & Heading \\
\hline Average & -33.942271329 & 18.852223497 & 131.2688 & -2.79335 \\
\hline
\end{tabular}

The main objective for this test was to determine the time it take for the GPS to be ready for georeferencing. A summary of the average time for the GPS to determine its position is 64.6 seconds and this is shown in Table 6. 
Table 6: GPS average deployment time.

\begin{tabular}{|l|l|}
\hline Deployment & Average time(s) \\
\hline Stellenbosch Square parking & 54 \\
\hline Mooiberge Farmstall & 50 \\
\hline Lyndoch Petrol station & 62 \\
\hline Dorp street and Die Boord & 20 \\
\hline Baden Powell Drive & 137 \\
\hline Average time & 64.6 \\
\hline
\end{tabular}

The average time was used as the time to geo-reference a system when using the GPS. The time was kept the constant for the GPS on all the geo-referencing comparisons.

\subsection{Summary of geo-referencing comparison}

Based on the geo-referencing comparison results, the average time to geo-reference a radar using the total station and prism was found to be $25 \mathrm{~min} 39$ s. Table 7 shows the total station deployment times and average.

Table 7: Total station average geo-referencing time.

\begin{tabular}{|l|l|l|}
\hline Deployment number & Time (sec) & Time \\
\hline Deployment 1 & 1760.4 & $29 \mathrm{~m}: 34 \mathrm{~s}$ \\
\hline Deployment 2 & 1395.6 & $23 \mathrm{~m}: 26 \mathrm{~s}$ \\
\hline Deployment 3 & 1353 & $22 \mathrm{~m}: 55 \mathrm{~s}$ \\
\hline Deployment 4 & 1633.8 & $27 \mathrm{~m}: 23 \mathrm{~s}$ \\
\hline Average & $\mathbf{1 5 3 5 . 7}$ & $\mathbf{2 5 m : ~ 3 9 s}$ \\
\hline
\end{tabular}

\subsection{Geo-referencing measurement results}

The geo-referencing data comparison between total station and GPS was collected in order to answer the main research question. It is important to note that the corner reflector was kept constant while the radar was moved around. The coordinates of the center point of the corner reflector were picked and used as the actual reference point for all deployments. To create local coordinates, the beacon was regarded as the origin therefore the coordinates of the corner reflector were offset from the beacon. Table $\mathbf{8}$ displays the recorded coordinates of the corner reflector based on the local coordinates format.

Table 8: Actual coordinates for corner reflector position.

\begin{tabular}{|l|c|c|c|}
\hline & X(North) & Y(East) & Z(Up) $[\mathrm{m}]$ \\
\hline Corner reflector coordinates & $\mathbf{- 3 3 . 9 6 4 4 4 4}$ & $\mathbf{1 8 . 8 3 5 1 8}$ & 142 \\
\hline $\begin{array}{l}\text { Actual coordinates(offset from beacon } \\
\text { coordinates) }\end{array}$ & $\mathbf{9 7 . 9 8 4 8 4 6}$ & $\mathbf{3 6 . 6 4 0 4 6 5 9 2}$ & 0.77 \\
\hline
\end{tabular}

A summary of the actual values, average values and difference between the actual and average for both the total station and GPS measurements are shown in Table 9 and Table 10. 
Table 9: Total station Summary of average values and difference between actual and average.

\begin{tabular}{|l|c|c|c|}
\hline \multicolumn{1}{|c|}{ Component } & Actual value & Average value & Difference between actual and average \\
\hline Latitude & $\mathbf{9 7 . 9 8 4 8 4 5 8 1}$ & $\mathbf{9 5 . 5 2 5}$ & $\mathbf{2 . 4 5 9 8 4 5 8 1}$ \\
\hline Longitude & $\mathbf{3 6 . 6 4 0 4 6 5 9 2}$ & $\mathbf{3 7 . 6}$ & $\mathbf{- 0 . 9 5 9 5 3 4 0 8}$ \\
\hline Height & $\mathbf{0 . 7 7}$ & -1.2 & $\mathbf{- 0 . 4 3}$ \\
\hline
\end{tabular}

Table 10: GPS Summary of average values and difference between actual and average.

\begin{tabular}{|l|c|c|c|}
\hline Component & Actual value & Average value & $\begin{array}{l}\text { Difference between actual } \\
\text { and average }\end{array}$ \\
\hline Latitude & $\mathbf{9 7 . 9 8 4 8 4 5 8 1}$ & $\mathbf{9 5 . 4 7 5}$ & $\mathbf{2 . 5 0 9 8 4 5 8 1}$ \\
\hline Longidude & $\mathbf{3 6 . 6 4 0 4 6 5 9 2}$ & $\mathbf{3 7 . 6 2 5}$ & $\mathbf{- 0 . 9 8 4 5 3 4 0 8}$ \\
\hline Height & $\mathbf{0 . 7 7}$ & $\mathbf{- 0 . 7 7 5}$ & $\mathbf{- 0 . 0 0 5}$ \\
\hline
\end{tabular}

The final comparison results can be represented on the matrix table as discussed in the earlier. The matrix system yielded the results shown in Table 11.

Table 11: Geo-referencing comparison matrix table

\begin{tabular}{|l|c|c|c|}
\hline \multicolumn{2}{|c|}{ Options } & \\
\hline Criteria & \multicolumn{1}{|c|}{ Weight } \\
\hline Accuracy & $\begin{array}{l}\text { Total station } \\
\text { Method }\end{array}$ & Automated GPS Method & \\
\hline Cost & $\mathbf{3}$ & $\mathbf{3}$ & $\mathbf{4 0 \%}$ \\
\hline Time & $\mathbf{5}$ & $\mathbf{4}$ & $\mathbf{2 0 \%}$ \\
\hline Effort & $\mathbf{3}$ & $\mathbf{5}$ & $\mathbf{2 0 \%}$ \\
\hline Reliability & $\mathbf{5}$ & $\mathbf{1 0 \%}$ \\
\hline & $\mathbf{5}$ & $\mathbf{5}$ & $\mathbf{1 0 \%}$ \\
\hline $\begin{array}{l}\text { Average weight for TS } \\
\text { Method }\end{array}$ & $\mathbf{3}$ & & \\
\hline $\begin{array}{l}\text { Average weight for GPS } \\
\text { Method }\end{array}$ & $\mathbf{4}$ & \\
\hline
\end{tabular}

\subsection{Equipment cost measurement}

The cost of the Spatial dual GPS is specified as 9126.00 Us dollars (USD) on the Advance navigation' website [19]. The price of the total station TS07 that is used by Reutech is listed as 8040.00 USD from Tiger Supplies [22]. The prisms and tripod are not included on the cost of the total station. The Leica GMP101 mini prisms that are used on the radar are priced at 180.00 USD [20]. The Leica tripod used is priced at USD 192 from Tiger Supplies [22]. This means the total cost of the total station equipment costs is 8412.00 USD. This shows that the total station is less expensive than the GPS. This means that the total station will obtain a score of five (5) on the matrix system. The score for the GPS is determined by calculating the score difference. The score difference is calculates as follows:

$$
\% \text { diff }=100 \%-\frac{\text { Lowest price }}{\text { Highest prices }} \times 100
$$




$$
\begin{gathered}
\% \text { diff }=100-\frac{8412}{9126} \times 100 \\
=\mathbf{7 . 8 2 \%}
\end{gathered}
$$

The percent result of the GPS is determined to be $7.82 \%$ and this result in a score of 4 on the matrix table.

\subsection{Reliability measurement discussion}

The reliability component of the matrix system was included to measure the number of attempts that each method requires in order to geo-reference the MSR. For all the deployments, geo-referencing was completed on first attempt for both the total station and GPS. This means that both these methods can score a value of five (5) on the matrix system.

\subsection{Effort measurement}

One of the components that were evaluated during the deployments was the effort it takes to geo-reference the radar on both systems. The total station method requires setting up the tripod, levelling the station itself and connecting the cable from the radar to the total station at least $10 \mathrm{~m}$ away from each other. Then the measurement of the prism and the reference point also took a lot of energy and effort to accomplish. The GPS method only required switching on and waiting for the coordinates to stabilize before using it and therefore there was not a lot of energy or effort required to accomplish geo-referencing. Given these scenarios, the GPS scored a value of five (5) on effort and the total station scored a value of one (1) on the effort category of the matrix system. 


\section{Conclusion}

The purpose of this research work was to integrate the GPS on the MSR, perform geo-referencing, deployment time, effort, cost and reliability comparison between the total station and the GPS when used for geo-referencing. To achieve the objective of this research, the Advance Navigation - Spatial dual GPS was chosen and integrated on the MSR using some of the principles that were obtained from the literature and the documentation provided by the manufacturer.

The first step was to confirm the GPS specifications by deploying the integrated system on five (5) different location. The main outcome for this test was to determine the time it takes for the GPS to get an average position. This was found to be $64.6 \mathrm{~s}$

The second step was to conduct a performance comparison between the total station and GPS. Four deployments were carried out for the comparison. The corner reflector was placed at a specific point and its coordinates were recorded. The radar was geo-referenced with both methods and the corner reflector was scanned to determine its relative position. The results of geo-referencing method were recorded and compared to the actual survey coordinates taken when setting up the corner reflector. In every deployment, the times for setting up the total station and geo-reference the system were recorded. The results of the total station deployment time were $25 \mathrm{~min} 39 \mathrm{~s}$ which is significantly longer than that of the GPS.

The accuracy results for all the four deployments on the matrix table showed that both the methods obtained an accuracy score of three (3) out of five (5) but if the results are closely looked at, it can be observed that the total station is more accurate than the GPS. The matrix table gave the average accuracy of the combined components of the coordinates.

Therefore, it can be concluded that it is possible to integrate the GPS on the movement and surveying radar. The comparison showed that the radar is able it be geo-referenced using the GPS. The accuracy of the GPS may differ depending on the location and satellites available. The results of the experiments also proved that the total station method of geo-referencing is more accurate than the GPS method and this compliments the work that was done by Chekole [15].

Acknowledgments: I express my deep sense of gratitude for Dr. Nkateko Mabunda for providing excellent support, valuable advice, support and guidance throughout my research. It is because of his assistance that this research has been successful. I would like to thank my family and friends who have supported me immensely throughout the duration of the research.

I would also like to thank my colleagues at Reutech, namely Nicholas Britz and Sharla Coetsee for their valuable input and helpful discussion. I appreciate their time and patience during the practical and writing of this paper.

I greatly acknowledge Reutech Radar Systems for providing me with the tools, time and bursary to study at the University of Johannesburg. 


\section{References}

[1] Kayesa, G. (n.d.). PREDICTION OF SLOPE FAILURE AT LETLHAKANE MINE WITH THE GEOMOS SLOPE MONITORING SYSTEM. In T. S. Metallurgy, International Symposium on Stability of Rock Slopes in Open Pit Mining and Civil Engineering (pp. 605-622).

[2] Reutech, R. S. (2013). Operator Manual Movement and Radar System. Stellenbosch: Unpublished confidential document.

[3] Sommer, S., \& Wade, T. (2006). A to Z GIS, An illustrated dictionary of Geographic Information Systems. 2nd ed. 89. Redlands: Esri Press.

[4] MacKinnon, T., \& Murphy, J. (2004). Introduction and Simple Guid to Using the Leica Total Station. COGS.

[5] Lackner,S, \& Lienhart, Werner. (2016). Impact of Prism Type and Prism Orientation on the Accuracy of Automated Total Station Measurements. Graz University of Technology, Steyrergasse 30/II, Graz, Styria, Austria: Institute of Engineering Geodesy and Measurement Systems (IGMS).

[6] Marshall, H., Coleman, P., Bolton, D., \& Cowell, A. (1998). GPS Applications at Optimum Colliery. The Journal of The South African Institute of Mining and Metallurgy, 127-134.

[7] Read, J., \& Stacey, P. (2009). Guidelines for Open Pit Slope Design. Collingwood: CSIRO Publishing: Australia.

[8] Kingsley, S., \& Quegan, S. (1999). Understanding radar systems. Mendham: SciTech Publishing Inc.

[9] Bagad, V. S. (2008). Radar Systems. India: Technical Publications Pune.

[10] Hoek, E., \& Bray, J. W. (1981). Rock slope engineering. London: The Institute of Mining and Metallurgy.

[11] Witwatersrand, U. o. (2013). Introduction to Mining Engineering . Johannesburg.

[12] Kovanič, L., Blišt’an, P., Zelizňaková, V., \& Palková, J. (2017). Surveying of Open Pit Mine Using Low-Cost Aerial Photogrammetry. Slovak Republic: Springer International Publishing.

[13]Leica Geosystems. (n.d.). Leica TS03/TS07 User manual.

[14]Feng, Y., \& Wang, J. (2008). GPS RTK Performance Characteristics and Analysis. Journal of Global Positioning Systems, 7.

[15] Chekole, S. D. (2014). Surveying with GPS, total station and terresterial laser scaner: a comparative study. Stockholm, Sweden.

[16] Mingqin , S., \& Xiuping , S. (2015). Study on Differential GPS Positioning Methods. Hangzhou, China: IEEE.

[17] Advance Navigation. (2017). Spatial Dual Reference Manual. Advance Navigation.

[18] Grimm, D. (2008). GNSS direction finding. Geodezija ir Kartografija, 34(3): 100-102.

[19] Advance Navigation. (2020, August 20). Retrieved from Advance Navigation: https://www.advancednavigation.com/product/spatialdual?gclid=CjwKCAjwh7H7BRBBEiwAPXjadlu5LFyoM1 x718YZAfDjusB5aBlOIxtXxakGor3oY2mcjANHswYchoCVHgQAvD_BwE 
[20] SUNBELT RENTALS. (2020, September 18). Retrieved from SUNBELT RENTALS: https://surveyequipment.com/leica-gmp101-mini-prism/

[21] SUNBELT RENTALS. (2020, September 18). Retrieved from SUNBELT RENTALS:

https://surveyequipment.com/leica-gmp101-mini-prism/

[22] Tiger Supplies. (2020, September 21). Retrieved from Tiger Supplies: Leica 868852 FlexLine TS07 Reflectorless Manual Total Station

[23]Zheng, Y.-T., Zha, Z.-J., \& Chua, T.-S. (2011). Research and Applications on Georeferenced Multimedia: A Survey." Multimedia Tools and Applications. Multimedia Tools and Applications , $77-98$.

[24] Linda, L. (2009). Georeferencing. MIT Press.

[25]Leica Geosystems. (n.d.). Leica TS03/TS07 User manual.

[26] Hackeloeer, A. (2014). Georeferencing: a review of methods and applications. Annals of GIS, 61-69. 